\title{
Measuring Affective-Cognitive Experience and Predicting Market Success
}

\author{
Hyung-il Ahn and Rosalind W. Picard, Fellow, IEEE
}

\begin{abstract}
We present a new affective-behavioral-cognitive $(A B C)$ framework to measure the usual cognitive self-report information and behavioral information, together with affective information while a customer makes repeated selections in a random-outcome two-option decision task to obtain their preferred product. The affective information consists of human-labeled facial expression valence taken from two contexts: one where the facial valence is associated with affective wanting, and the other with affective liking. The new "affective wanting" measure is made by setting up a condition where the person shows desire to receive one of two products, and we measure if the face looks satisfied or disappointed when each of the products arrives. The "affective liking" measure captures facial expressions after sampling a product. The ABC framework is tested in a real-world beverage taste experiment, comparing two similar products that actually went to market, where we know the market outcomes. We find that the affective measure provides significant improvement over the cognitive measure, increasing the discriminability between the two similar products, making it easier to tell which is most preferred using a small number of people. We also find that the new facial valence "affective wanting" measure provides a significant boost in discrimination and accuracy.
\end{abstract}

Index Terms - affective liking, affective wanting, behavioral experiment, beverage taste test, cognitive liking, cognitive wanting, consumer research, customer experience, decision utility, facial valence, market success prediction, marketing research, new product test, self-reported emotion

\section{INTRODUCTION}

$\mathrm{M}$ easuring customers' experience to predict their preference toward new products or services is difficult: today's state-of-the-art methods use focus groups and cognitive self-reports where respondents are asked about what they like or want. These methods, while often employed in marketing and consumer research, are not reliable predictors of the ultimate customer preference. Considerable doubt has been cast over the validity and reliability of self-report measures of emotional experience [1],[2],[3]. Self-report measures often capture cognitive and subjective elements of overall emotional experience after a product evaluation (e.g., what you think you should say you like or want) but may or may not capture actual momentary feelings during the evaluation. Each respondent's self-reports tend to be answered using his or her own relative scale and reference point. Also, they are likely to draw on a distorted and biased memory of the actual emotional experience often relying on a peak (intense-emotion) or end moment during the experience [4].

To allow a fuller representation of customer experience to be used in making important decisions about new products or services, we we think it is important to move beyond self-report cognitive-only measures. This paper presents a new methodological framework for naturally eliciting and

- Hyung-il Ahn is with IBM Research - Almaden, San Jose, CA 95120. E-mail: hiahn@us.ibm.com.Thisworkwas mainlydone when he was a doctoral candidate at the MIT Media Lab, Cambridge, MA 02139.

- R. W. Picard is with the MIT Media Lab, Cambridge, MA 02139 and also with Affectiva,Inc, Waltham, MA 02452.E-mail:picard@media.mit.edu.

Manuscript received (insert date of submission if desired). Please note that all acknowledgments should be placed at the end of the paper, before the bibliography. reliably measuring respondents' expressions of their intuitive feelings about products or services. The new affectivebehavioral-cognitive $(A B C)$ framework can systematically and efficiently gather affective measures (facial expression valences), behavioral measures (physical number of choices, consumed amounts, evaluation times), and cognitive selfreports ("how much do you like this product" and "how likely are you to buy this product"). The state of the art methods in common practice today usually employ only cognitive self-report methods. Our method includes self-report questions for comparison, but goes beyond this.

While self-report questions may ask about affective experience, we group them under cognitive measures because filling out a questionnaire is fundamentally a process of thinking about and reflecting on one's experience and feelings. The $\mathrm{ABC}$ framework offers both a practical new method to elicit the three types of measures and a computational method to combine them to infer respondents' preference. In addition, the framework can be used to predict the market success/failure of new products or services. Standard practice today usually makes any such predictions by looking at the value of one or more self-report measures and trying to relate it to sales. In the real-world case examined here, the $\mathrm{ABC}$ framework makes these predictions significantly more accurately than cognitive measures do alone.

People may sometimes require multiple experiences of a product before they actually notice its qualities, for better or worse. For example, people tend to prefer a sweeter beverage in one-sip blind tests, as often called the "Pepsi Paradox" [5], but multiple sips or drinking a whole can of sweet beverages can be too sweet and an overall bad experience [6]. When Coca-Cola invented "New Coke" in 1985, they 


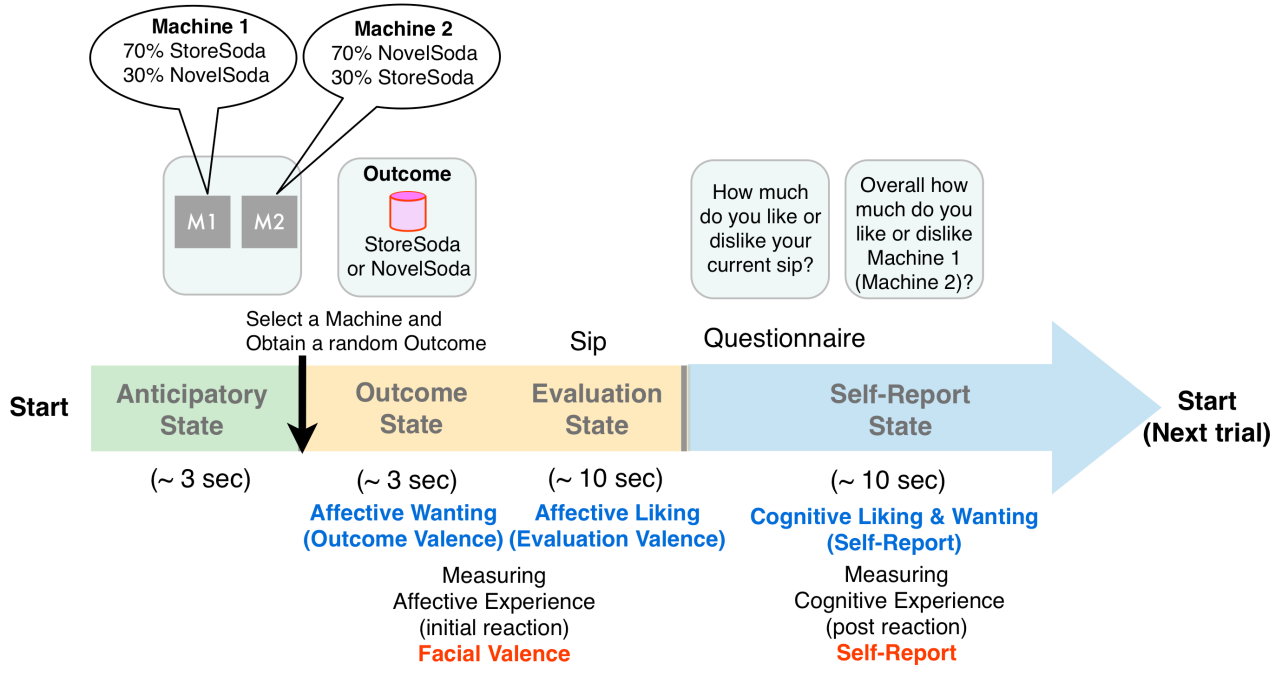

Fig. 1. The sequence for each trial. Each trial involves selecting a machine, sipping the beverage, and answering questionnaire items. At two key points each trial, facial valences are measured for wanting and liking. Each respondent repeated this sequence for 30 trials, taking a total of 30 sips.

conducted one-sip blind tests for focus groups. In those tests, people said they liked New Coke better, but after it went to the marketplace it did not sell as well. "New Coke" was sweeter than "Classic Coke" [7]. To avoid this problem and in order to more accurately reflect market conditions, our framework begins by addressing two principles in order to elicit accurate affective information: (1) a respondent should experience each product for multiple trials, because their liking and wanting of products can change after initial impressions; and (2) a respondent should be made comfortable to freely express both wanting and liking, positive and negative.

The latter point is particularly important because most cultures have contexts in which it is inappropriate to show certain kinds of facial expressions. For example, most children in America are taught to not show people negative faces after sampling food that they do not like. Thus, American adults often suppress facial expressions of disliking.

Suppression of the embodied expression may further interfere with the feeling and reporting of an emotion [8]. However, most people in America have not been taught to suppress negative facial expressions toward computers or machines. This contextual impact on emotional expression is one thing we exploit in our framework for comfortably eliciting affective information.

In our framework, in order to elicit a respondent's affective-behavioral-cognitive responses in a trial-by-trial manner, we introduce a tool from machine learning that was adapted from casinos - a two-armed bandit [9] - a slot machine with two arms, either of which you can choose to pull, and each of which gives a different random distribution of outcomes. The two stochastic outcome distributions are initially unknown to respondents. Thus, initially they explore pulling both "arms" to see what both do. Over time, they learn that the left arm favors giving them one of the two products, while the right arm favors the other. At that point, we expect to see a behavioral bias toward pulling the arm of the most favored product, if they have a strong preference.
We conducted an examination of the validity and effectiveness of our methodology by conducting a real-world beverage test in partnership with a famous consumer product company. Two test beverages from an international soda manufacturer, called FamousBrand in this paper, were selected because they were considered favorable and similar to each other in early tests by FamousBrand using standard market research (cognitive self-report only) measures; however, they had very different market selling results. One, "StoreSoda," performed in the top $25 \%$ of the market, while the other "NovelSoda," performed in the lower 40\%, despite that people said NovelSoda was as good as StoreSoda in pretesting. More specifically, StoreSoda is a cola product with vanilla flavors that has been in the North America market for the last 10 years. NovelSoda was a cola product with tropical fruit flavors that was released and discontinued in the same year, a year that StoreSoda was also on the market. Both products had the same internationally-renown parent brand who performed comparable marketing efforts for both products.

In the experiment we created a new version of the twoarmed bandit: we map each arm to a soda machine that provides respondents with one of two outcomes: a StoreSoda or NovelSoda. Each time a soda is provided as the outcome in a trial, respondents are asked to sip the soda. Each machine exhibits a different pattern of outcomes with regard to which soda is provided. For example, one machine gives StoreSoda with a higher probability, say $70 \%$ and NovelSoda with $30 \%$, while the other machine gives NovelSoda with $70 \%$ probability and StoreSoda with $30 \%$. The respondent is essentially able to figure out which machine provides his or her favored beverage more frequently and will select that machine more often. The use of the two-armed bandit addresses the first principle that respondents try a sip of each product many times. Even if they stick with the machine more likely to give their favored product, the randomness of outcomes from each machine prevents their anchoring to that product, e.g., if they like StoreSoda, and favor the ma- 


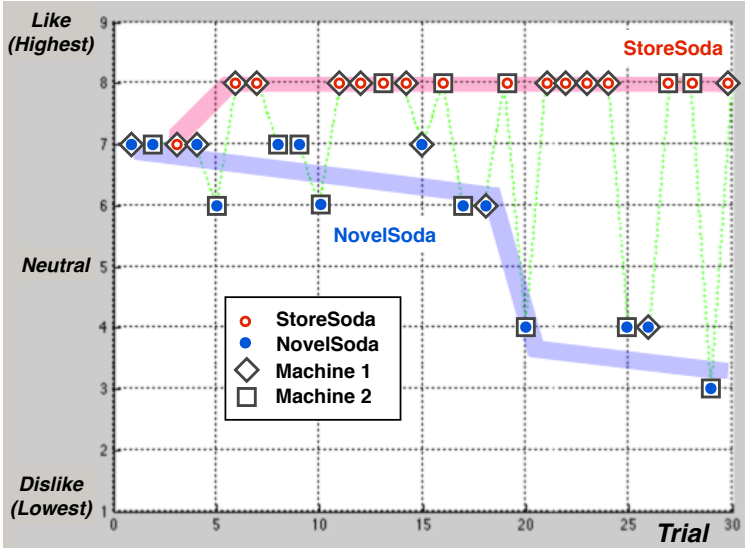

(a)

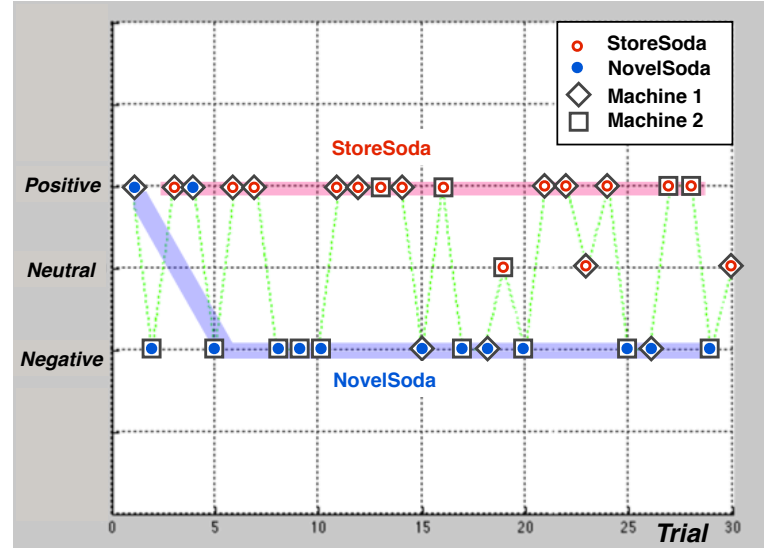

(b)

Fig. 2. (a) Self-reported liking (questionnaire) over 30 sips (trials), where on each trial a machine is selected and gives one of two beverages to sip. Selfreport is initially high for both sodas, and falls off for NovelSoda after 20 trials. (b) Facial valence for the same person over the same 30 trials. Facial valence is initially positive for both sodas, yet becomes negative for NovelSoda after 5 trials. Also, the thick transparent red and blue lines show the trend for each soda.

chine more likely to give it, they still occasionally have to try another sip of NovelSoda. Also, the computer-presented bandit tasks involving an unknown outcome may help more reliably reveal natural feelings about products, addressing the second principle above. That is, the method we propose and employ in our study further enables the researcher to naturally elicit not only "affective liking" (evaluative/hedonic/sensory affect) but also "affective wanting" (anticipatory/motivational affect) - a kind of motivation that is believed to be very significant in determining market success [10], [11], [12], [13],[14],[15].

More specifically, we can infer the valence of the respondent's wanting of a product (positive or negative) from facial expressions immediately after they have made a choice and received a random outcome from each trial. For example, consider a person who, after several trials, favors StoreSoda, wants StoreSoda, and selects the machine he or she has determined is more likely to give that choice. When the machine provides StoreSoda, if we see a facial expression that indicates satisfaction, then we can infer that there was likely positive affective wanting for that beverage. Similarly, when the machine provides the unwanted outcome and elicits an expression indicating disappointment, we can infer that there was negative affective wanting, i.e., the respondent did not want that particular soda. We found respondents to be quite expressive at showing satisfaction or disappointment during this "outcome" measurement timewindow in each trial (i.e., right after obtaining an outcome), suggesting that this environment was a comfortable one for them to make both positive and negative facial expressions, addressing the second principle. Our results presented below suggest that this novel measure of affective wanting provides a significant boost in discrimination and accuracy.

We define three states respondents experience in each trial as follows. Respondents experience an anticipatory state before and while choosing a machine, an outcome state right after obtaining an outcome, and an evaluation state while trying and evaluating the obtained outcome. Fig. 1 shows the sequence for each trial. In each trial we typically observe people's anticipation (e.g., autonomic arousal) before selecting an option, immediate satisfaction/disappointment from facial expressions with the obtained outcome, and liking/disliking from facial expressions while evaluating the outcome. Importantly we distinguish the "affective wanting" responses (called outcome facial valence in this paper) from the "affective liking" responses (called evaluation facial valence in this paper). The former is a response to the machine delivering the beverage that the person did or did not want, before sipping the beverage; the latter is a response to the actual sip.

Affective liking for different outcomes can be measured more directly, during respondents' actual evaluations of the outcomes. When a person sips the outcome sample beverage and likes the taste, he or she is likely to show positive affective liking (e.g., looking pleased, smiling, licking the lips). Otherwise, the person is likely to show negative affective liking (e.g., looking displeased, frowning, showing disgust) [10], [16], [17]. We also have a neutral facial valence condition if none of these are observed. Our method also measures how expressions change over several encounters with the same product - perhaps starting off positive, maintaining that state or fading to neutral, or changing to negative after repeated trials.

To briefly explain our protocol, Fig. 2(a) shows a sample of 30 trials of data collected from the actual human experiment for a representative respondent, where on each trial the respondent selects a machine, it dispenses a soda, the respondent takes a sip and self-reports the liking for that sip.

First, in Fig. 2(a) we see the respondent's self-reported liking was high initially for both sodas. A traditional protocol that does not give a respondent an adequate number of opportunities to experience a product may conclude too quickly that their feelings are positive toward both products. In fact, the traditional protocol used by FamousBrand concluded that NovelSoda should go to market, and should sell 
as well as StoreSoda. Consequently, valuable resources human, energy, and more - were expended manufacturing, packaging, marketing, and trucking the product to stores, and then later trucking the unsold product back from the stores.

It took 20 sips before this respondent self-reported disliking NovelSoda. Fig. 2(b) shows facial valence (positive, neutral or negative) over trials for the same respondent. Facial valence was initially positive, but after 5 sips shows negativity for NovelSoda. Here, the affective measure of facial valence was faster to communicate preference than the cognitive measure of self-report, and was also more natural for the respondent to use than trying to figure out how to assign a number to his changing feelings. Also, here the entire interaction was with the computer, not with a person, so the facial valence can be more directly related to the experience with the product and with the computer's question about the product, and is less likely to be related to interaction with the experimenter, which can lead to social desirability bias [18].

The rest of this paper is presented as follows: Section 2 briefly highlights concepts related to affective wanting and liking in the human decision-making literature. Section 3 describes our experiment methods, cognitive, affective and behavioral measures employed in our real-world experiment, and basic observation results. Section 4 presents two main sets of analyses and the results. Finally, Section 5 gives conclusions and discussions. As far as we know, this framework is the first to systematically elicit and integrate both people's affective wanting and liking, together with behavioral choice and self-reported cognitive wanting and liking during natural decision-making and evaluation processes, describe their preferences in terms of these multi-modal measures, and use all of this information to computationally predict market success/failure of new products or services.

\section{CONCEPTUAL BACKGROUND}

Our conceptual framework draws from important findings in the human decision-making literature of psychology, behavioral economics and affective neuroscience. We briefly highlight common or nearly similar concepts from these fields and associate the concepts with the terms in this paper.

\subsection{Wanting and Liking}

Affective neuroscience research points out that motivational/anticipatory aspects or "wanting" can be distinguished from evaluative/hedonic/sensory aspects or "liking" in human decision-making and learning processes [19], [11], [12], [14]. This means that we need to distinguish different meanings of the term "utility", which has been used to represent multiple concepts related to wanting and to liking in the history of psychology and economics. Kahneman distinguished "experienced utility" from "decision utility" [1],[4] where the former concept relates to liking and the latter to wanting. Experienced utility is associated with sensory and hedonic properties of the outcome of a choice, and arises from evaluation of our experience of the outcome [1], e.g. the sip of the beverage. Decision utility is associated with motivational properties of the future expected outcome of a choice, and relates to the degree to which a choice is selected, e.g. how much the person selects one soda machine relative to the other.

\subsection{Cognitive Wanting and Affective Wanting}

Multiple motivational systems systematically influence human decision behavior, including both cognitive (expected, deliberative, goal-directed, reflective, conscious, controlled) and affective (anticipatory, intuitive, habitual, reflexive, unconscious, automatic) processes [13],[20], [21], [22], [23], [24],[25],[26],[27]. Note that the terms "cognitive" and "affective" in our framework are generally used to indicate the properties of affective and cognitive processes detailed above inside the parentheses, even though these components may not always be cleanly separable in practice. According to Berridge and Robinson [12], wanting can be dissociated into the unconscious core process of incentive salience 'wanting' (associated with "affective wanting" in this paper) and the conscious subjective awareness of cognitive wanting (associated with "cognitive wanting" in this paper). The two differ both psychologically and in their brain substrates: affective wanting is a cue-triggered unconscious motivation mediated by mesolimbic dopamine systems, while cognitive wanting is an explicit cognitive expectation about future experience mediated by cortical systems.

\subsection{Cognitive Liking and Affective Liking}

Liking can be dissociated into the unconscious core process of 'liking' (associate with "affective liking" in this paper) and the conscious subjective awareness of cognitive liking (associated with "cognitive liking" in this paper). Moreover, note that, the neural substrates of affective liking are separate from those of affective wanting in the human brain [12].

\section{EXPERIMENT}

Our experiment presented the two test beverages (StoreSoda and NovelSoda from FamousBrand) in the same way, with identical controlled temperature, glasses, quantity, etc., and in the same modern test facility that FamousBrand usually conducts their tests. FamousBrand's test facility recruited "sweet cola drinkers" as respondents in their usual way. The tests took place on summer days with warm, sunny weather in a comfortable modern air-conditioned facility.

\subsection{Methods}

Respondents: Thirty-nine respondents aged 18-49 years (17 men, 22 women) participated in this experiment. Each respondent was asked to fill out an informed consent form and this experimental protocol was pre-approved by the MIT Committee on the Use of Humans as Experimental Subjects. Sensors: The computer in front of the respondent includes a tiny built-in webcam taking video of his or her face. Facial expressions were measured during the entire experiment, a time period of 30 minutes on average.

Tasks: After respondents were settled in the testing room, the experimenter left the room and each respondent was left alone, and asked to follow the instructions displayed on the computer. In addition to tasting beverages and answering questions, they were instructed to "try and figure out which 
machine more often directs you to the beverage you enjoy more and select that machine more often." Respondents were not given any product names but were exposed to the famous brand logo and given code labels (135 or 246) that were randomly assigned to each beverage before the experiment. Fig. 3 shows the general setup in the experiment.

Each respondent had 30 trials: Each trial, composed of the steps in Fig. 1, began with the respondent selecting one of the two machines, M1 on the left, or M2 on the right side of the computer screen. The machine indicated an outcome beverage (presenting 135 or 246). Then, the respondent sipped the outcome beverage from the side of the selected machine, and self-reported his or her cognitive liking value (answering the question "How much do you like or dislike your current sip?").

The respondents were not told this: One machine provided StoreSoda $70 \%$ of the time and NovelSoda $30 \%$ of the time, and the other provided $30 \%$ StoreSoda and $70 \%$ NovelSoda. Below we refer to Machine $S$ as the machine more likely to provide StoreSoda, and Machine $N$ as the one more likely to provide NovelSoda. For half of the respondents Machine $S$ was on the left side and Machine $N$ on the right side; the order was swapped for the other half of respondents. The names Machine $\mathrm{S}$ and Machine $N$ were not revealed to participants. Participants saw only "Machine 1" on their left and "Machine 2" on their right.

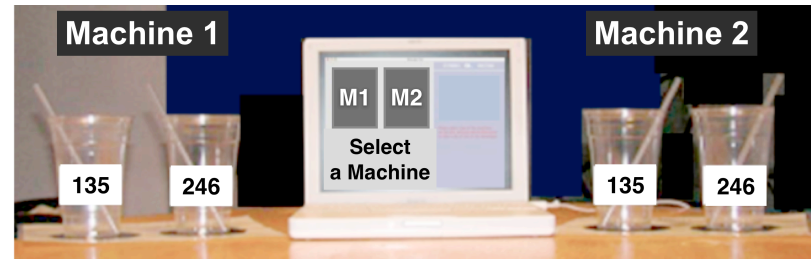

Fig. 3. Experiment Setup

\subsection{Measures and Observations}

Affective measures (facial valence): affective liking value, affective wanting value

We measure the affective liking value by recording the evaluation facial valence, viz., the facial valences during and immediately after tasting a product. Each respondent gets 30 affective liking values, one for each of the 30 trials.

We measure the affective wanting value by recording the outcome facial valence. When respondents get the option they want from a soda machine, they tend to show positive facial valence (or satisfaction). When they obtain the undesired outcome, they tend to show negative facial valence (or disappointment). This positive or negative facial response (satisfaction or disappointment) immediately after obtaining an outcome is called the outcome facial valence. The facial expression in this outcome state (i.e., immediately after the machine tells them which soda they will get to sip) provides information on the respondents' wanting in that trial; thus, for each respondent, we get 30 values of affective wanting. ${ }^{1}$

\footnotetext{
In our analysis the affective wanting measure was considered from the beginning of the experiment, after we confirmed that we found no outcome facial valence on the first trials of each soda.
}

We hired two human facial-affect recognition coders who scored above average on the Reading-the-Minds-in-the-Eyes revised test [28], and instructed them to label the outcome facial valence (= affective wanting value) and the evaluation facial valence (= affective liking value) for each trial. We asked each coder to label the outcome and evaluation facial valences into positive (1) / neutral (0) / negative (-1) responses. Smiling, nodding, looking pleased, satisfied, or licking the lips were rated positive, while frowning, shaking the head, showing disgust, or otherwise looking displeased or disappointed were rated negative $[10,16,17]$. Lack of any expression, or when the two coders disagreed, resulted in a neutral score.

On each trial $t$ we obtain the affective wanting value, $F V_{W}(t)$, as outcome FV ( 1 if positive, 0 if neutral, -1 if negative) and the affective liking value, $F V_{L}(t)$, as evaluation FV ( 1 if positive, 0 if neutral, -1 if negative). Also, the combined "affective value," $F V(t)$, also called facial valence $(\mathrm{FV})$ value, is modeled by $F V(t)=a_{L} F V_{L}(t)+a_{W} F V_{W}(t)$ using $a_{L}=a_{W}=0.5$. With more product tests and real-word market success/failure data in the future these weight values could be trained using machine learning. It might be that temporally-adapted weights or other adaptations would further improve the findings we obtain here. Because we want to avoid overfitting given we do not have but two real-world sets of marketing data, we start with assuming equal weights here. Learning of optimal weights will be future work when there are more data sets. For each respondent, we form the average affective liking (or wanting) value of each beverage simply as the average value of all $F V_{L}(t)$ 's (or $F V_{W}(t)$ 's) over trials on which that beverage was evaluated.

Affective measure observation: Due to MacBook video recording errors for five respondents, we collected a total of 34 valid recordings for the facial valence analysis. The evaluation of the two human coders' agreement is described below.

We divided respondents into two groups: the "FV expressive group" and the "FV non-expressive group". The FV expressive group is defined to be the respondents who showed valenced outcome or evaluation FVs at least once over all 30 trials. The respondents in the FV non-expressive group showed no facial expressions (or very few indistinguishable micro-expressions) during the task. Half of the 34 respondents for whom we have video belong to the FV expressive group. For this group $(\mathrm{N}=17)$, the two human coders had "moderate agreement" in terms of Cohen's Kappa: Observed agreement $=70.9 \%$ (718 out of $1020=17$ respondents $\times 30$ trials $\times 2$ FVs), Cohen's kappa $=0.5014$ (meaning "moderate agreement"). We accepted positive or negative codes when the two human coders agreed with each other; otherwise we considered the responses neutral. The FV analysis results below are based on the FV expressive group.

Cognitive measures (self-report): cognitive liking value, cognitive wanting value

Kahneman [1] suggested measuring "instant utility" as a measure of liking by asking a person to self-report immediate liking every time window or trial. We call such a selfreport measure the cognitive liking value. The question, 
"How much do you like or dislike your current sip?" was asked after every sip to obtain the respondent's liking on a 1 to 9 scale. The answers were converted to -4 to 4 for analysis: Like it extremely $(=4)$, Like it very much $(=3)$, Like it moderately $(=2)$, Like it slightly $(=1)$, Neither like nor dislike $(=0)$, Dislike it slightly $(=-1)$, Dislike it moderately (= $2)$, Dislike it very much $(=-3)$, Dislike it extremely $(=-4)$. This self-reported cognitive liking value is denoted $S R_{L}(t)$ on trial $t$ and always corresponds to the beverage that was sipped (only one beverage is sipped on each trial).

To measure the cognitive wanting value for each product, every $(5 n-1)$ th trial $(n=1, \ldots, 6)$ we asked the respondent to self-report the answers to two questions, "Overall how much do you like or dislike Machine 1 (Machine 2)?" in addition to the question to ask about their immediate liking. The respondent answered these for both machines before he or she selects one of the two. The two questions for both machines were on a 1 to 9 scale, and converted to -4 to 4 for analysis. Then, we approximate the cognitive wanting of each product by the linear combination of the two responses using as weights the estimated observed probability of each option to have provided the product as an outcome. In other words, cognitive wanting value $S R_{W}(b, t)$ of beverage $b$ ( $1=$ StoreSoda or $2=$ NovelSoda) on trial $t$ is modeled by $S R_{W}$ $(b, t)=P_{t}(b \mid m=1) S R_{M}(m=1, \mathrm{t})+P_{t}(b \mid m=2) S R_{M}(m=2, \mathrm{t})$ where $S R_{M}(m, t)$ is the machine liking value for machine $m$ $(=1,2)$ on trial $t$, and $P_{t}(b \mid m)$ is the estimated observed probability of machine $m$ to have provided beverage $b$ on trial $t$. Note that every five trials the two machine-related values are used to update the cognitive wanting value $\left(S R_{W}\right)$ of each beverage $b$. This indirect measure should be less influenced by cognitions or social pressure of "what I'm supposed to want" than the direct measure of asking the person explicitly which beverage they most want, although it might be interesting to compare this direct question in future work. The combined self-reported "cognitive value" $(S R(t))$ is modeled by $S R(t)=c_{L} S R_{L}(t)+c_{W} S R_{W}(b, t)$ where $c_{L}=c_{W}$ $=0.5$ and $b$ is the evaluated beverage on trial $t$. For each respondent, the average cognitive liking (or wanting) value of each beverage is the average value of all $S R_{L}(t)$ 's (or $S R_{W}(b, t)$ 's) over trials on which that beverage was evaluated.

Finally, respondents indicated their "after-the-test" beverage preference on a final questionnaire given after all trials were completed.

Cognitive measure observation: Comparing after-the-test self-reported beverage preferences, the ratio of \#StoreSoda favorers: \#NovelSoda favorers: \#No preference was 20:18:1. Pooling for both beverages, there was no significant difference between the average cognitive liking value and the average cognitive wanting value of each beverage over all respondents $(M=1.49$ vs. 1.26 ; within-subjects, $T(77)=1.65$, $N S$ ). It should be noted that there were significantly more positive responses than negative ones in terms of cognitive liking $(M=21.7$ vs. 3.44 ; within-subjects, $T(38)=9.33, p<$ $.001)$, and also in terms of cognitive wanting $(M=22.6 \mathrm{vs}$. 5.41; within-subjects, $T(38)=8.07, p<.001)$.

These findings provided important confirmation: they show that our group of testers, while different from those in FamousBrand's original study, were similar in that they indicated that both products were liked and wanted. The overall questionnaire items suggested that NovelSoda should have as good a chance of success as StoreSoda in the marketplace, all other conditions being equal.

In terms of the average cognitive liking values, respondents showed no significant difference between StoreSoda and NovelSoda ( $M=1.54$ vs. 1.43 ; within-subjects, $T(38)=$ $0.25, N S$ ). Also, the average cognitive wanting values did not indicate any significant preference over respondents $(M=1.23$ vs. 1.31 ; within-subjects, $T(38)=-0.59, N S)$. In like manner, the average cognitive values (from the combined measure of cognitive liking and wanting) showed no significant difference over respondents $(M=1.39$ vs. 1.38; within-subjects, $T(38)=0.04, N S$ ). All of these nonsignificant differences in the average liking, wanting, and combined values of beverages indicate that the traditional cognitive measures self-reported during trials are not providing fine discrimination for telling which beverage is preferred, nor are they providing differences that FamousBrand saw in the actual marketplace where StoreSoda sales were in the top $25 \%$, while NovelSoda performed in the bottom $40 \%$.

A subset of our ABC method is thus directly comparable to the traditional cognitive-only based methods of self report. Moreover, we found no significant differentiation between the cognitive liking values reported after first sipping StoreSoda or first sipping NovelSoda ( $M=1.79$ vs. 1.64; within-subjects, $T(38)=0.47, N S)$. Confirming the reasoning behind the first principle above, people needed time to be able to articulate their preferences: the first-sip preference ratio showed more persons who did not decide their beverage preference (the ratio of $\#$ favorers $=15: 15: 9$ ), compared to the after-the-test preference ratio $(20: 18: 1)$.

Behavioral measures - behavioral wanting and behavioral liking: For a behavioral measure of wanting, we recorded respondents' machine choices and each machine's ratio of giving the two sodas. For a behavioral measure of liking, we measured the consumed amount of each beverage.

Behavioral measure observation: Behaviorally, people tended to select Machine $\mathrm{N}$ more (whether it was on the right or left), which is likely to give the more novel beverage, NovelSoda: $(M=46.4 \%$ vs. $53.6 \%$ (machine $\mathrm{S}$ vs. machine $\mathrm{N}$ ); within-subjects, $T(38)=-1.63, p=.055)$. Whereas "afterthe-test" NovelSoda favorers chose Machine N significantly more often $(M=43.1 \%$ vs. $56.9 \%$; within-subjects, $T(17)=-$ $3.30, p<.01)$, "after-the-test" StoreSoda favorers showed no significant preference between machines $(M=48.8 \%$ vs. $51.2 \%$; within-subjects, $T(19)=-0.31, N S$ ). Also, while respondents overall did not show any significant difference in the amount consumed per sip between the two sodas $(M=$ 0.44 vs. $0.44 \mathrm{oz} / \mathrm{sip}$; within-subjects, $T(38)=0.0, N S$ ), those who preferred StoreSoda after the test consumed more StoreSoda per sip than NovelSoda per $\operatorname{sip}(M=0.43$ vs. 0.35 $\mathrm{oz} / \mathrm{sip}$; within-subjects, $T(19)=2.45, p<.05)$.

Interestingly, respondents tended to consume more beverage per sip when they obtained an unexpected outcome from a particular trial - NovelSoda from Machine $\mathrm{S}$ or StoreSoda from Machine N - compared to when they ob- 
tained an expected outcome - StoreSoda from Machine S or NovelSoda from Machine N (0.53 oz/sip vs. $0.39 \mathrm{oz} / \mathrm{sip}$, within-subjects, $T(38)=4.96, p<.001)$. This finding can be considered consistent with other findings that uncertainty is a mild stressor and people tend to eat or drink more when stressed [29, 30].

Overall, for this group of size 39, while there were some significant differences in behavior, machine selection related more significantly to exploring a novel beverage than to choosing the machine providing their after-the-test preferred beverage. Beverage consumption quantity related more to gulping bigger sips after an unexpected outcome than to consuming more of the after-the-test preferred beverage. The behavioral results thus did not show strong differentiation among the beverages.

\section{ANALYSES AND RESULTS}

In this section we present two main analyses: first, the affective and cognitive discriminabilities of individual beverage preference, and second, the prediction of market success/failure of beverages.

\subsection{Analysis 1: Affective and Cognitive Discriminabilities of Individual Beverage Preference}

We define cognitive value (self-report (SR)) on each trial as the linear combination of cognitive wanting and cognitive liking during the trial. This is the value shown in Fig 2(a). In like manner, we define affective value (facial valence (FV)) on each trial as the linear combination of affective wanting (outcome FV) and affective liking (evaluation FV) during the trial. This is the value shown in Fig 2(b). In the study here we use equal weights for both linear combinations.

Often, two products are very similar and peoples' ratings do not differ significantly. This is a common problem requiring a company to recruit huge groups of respondents before a statistically significant difference is obtained. A better measure will, for a fixed number of respondents, obtain a greater discriminability "d-prime" $\left(d^{\prime}\right)$, estimated as $d^{\prime}$ $=Z$ (sensitivity) $-Z(1-$ specificity $)$ where $Z(p), p \in[0,1]$ is the inverse of the standard cumulative normal distribution, sensitivity $=$ true positive rate, and $1-$ specificity $=$ false positive rate $=1-$ true negative rate [31]. As the discriminability (i.e., between the most favored beverage vs. the least favored beverage) becomes larger, it is easier to discriminate the two beverages. Thus, a measure (SR or FV) with greater $d^{\prime}$ is more conducive to discriminating differences between two beverages, even if they are similar sweet sodas as in the real-world case we studied. Note that under the assumption that the two observed distributions are normal with the same standard deviation, the estimated discriminability $d^{\prime}$ can be related to Cohen's $d$ (= the difference between two means divided by a standard deviation for the observed data), which is frequently used in estimating the sample size to obtain the desired statistical power or significance level [32].

Regarding the cognitive and affective values, we make the following hypotheses:

H1: Cognitive value and affective value do not have high correlation. That is, affective value carries different information from cognitive value. Specifically we expect people to be more positive in the SR measure than in the FV measure.

H2: In terms of discriminability for distinguishing the most favored beverage from the least favored beverage, $d_{\text {SR }}^{\prime}$ ( $d^{\prime}$ from SR) and $d_{\mathrm{FV}}^{\prime}\left(d^{\prime}\right.$ from FV) will not be significantly correlated; thus, there will be respondents whose most/least favored beverages can be easily detected in terms of FV but not in terms of SR and vice versa.

We need to define an objective overall preference between the two beverages in order to assess the accuracy of the proposed method, but there is no flawless measure of preference known to be obtainable in a short-term study. In this study we accord special status to the preference stated after the maximum number of trials (30 in this study). We define the after-the-test most (or least) favored beverage as the beverage the respondent ultimately said that they preferred (or did not prefer) on their after-the-test questionnaire after all (30) sips have been experienced (total, combining sips from the two beverages). While this is a cognitive measure and is not perfect as such, it is a common choice in the industry, we have it for all respondents, and it at least occurs after maximal experience with both products within the study.

TABLE 1

COUNTS OF TRIALS WITH OUTCOME FV (VALENCED, NEUTRAL) AND EVALUATION FV (VALENCED, NEUTRAL) IN THE FACIALLY EXPRESSIVE GROUP OF RESPONDENTS $(\mathrm{N}=17)$

\begin{tabular}{|c|c|c|c|}
\hline & $\begin{array}{c}\text { Outcome FV } \\
\text { (Pos or Neg) }\end{array}$ & $\begin{array}{c}\text { Outcome FV } \\
\text { (Neutral) }\end{array}$ & Total \\
\hline $\begin{array}{c}\text { Evaluation FV } \\
\text { (Pos or Neg) }\end{array}$ & $44(8.6 \%)$ & $110(21.6 \%)$ & $154(30.2 \%)$ \\
\hline $\begin{array}{c}\text { Evaluation FV } \\
\text { (Neutral) }\end{array}$ & $78(15.3 \%)$ & $278(54.5 \%)$ & $\begin{array}{c}356 \\
(69.8 \%)\end{array}$ \\
\hline Total & $122(23.9 \%)$ & $388(76.1 \%)$ & $\begin{array}{c}510(100 \%= \\
17 \text { people } \times \\
30 \text { trials })\end{array}$ \\
\hline
\end{tabular}

Affective liking and affective wanting show differences: Table 1 shows how many times outcome FV and evaluation FV happened together, separately, or not at all (staying neutral = not showing any valenced expression) in all 30 trials for the expressive group $(\mathrm{N}=17)$. We checked individual trials where any facial expressions were made. In 44 trials the person showed both evaluation and outcome facial valences. In 188 trials the person showed only a response from either evaluation FV or outcome FV. In 43 of the 44 trials, the outcome and evaluation FVs had the same valence (both positive or both negative), showing strong consistency within a trial. While people were more likely to show a positive or negative evaluation FV after sipping, about $24 \%$ of the total trials included a positive or negative outcome FV that occured after the machine indicated which beverage they would have to sip, a new measure we propose as an indication of wanting.

Following Berridge's dissociation between implicit 'wanting' and implicit 'liking', we expect that the two affective measures will show some level of independence across 
trials. Also, note that the sensor measurements of affective wanting and liking have no measurement dependence between each other. We thus examined, and indeed found that, pooling for both beverages, there was a significant difference between the average affective liking (across all trials) and the average affective wanting (across all trials) over the facially expressive group, $(M=-0.12$ vs. -0.02 ; withinsubjects, $T(33)=2.08, p<.05)$. We also found the relative independence of these measures across the whole test (30 trials). The affective liking (evaluation FV) measure had significantly more negative responses than positive $(M=6.12$ vs 2.94; within-subjects, $T(16)=2.46, p<.05)$, and showed significantly more negative responses than the affective wanting (outcome FV) measure $(M=6.12$ vs. 3.76; withinsubjects, $T(16)=1.85, p<.05)$.

Difference between cognitive value (SR) and affective value $(F V)$ : We examined data related to hypothesis $H 1$, whether affective value (facial valence (FV)) and cognitive value (self-report (SR)) reveal the same or different information. In each trial a respondent showed one of nine different SR and FV combinations (Table 2: positive/neutral/negative SR crossed with positive/neutral/negative FV). Responses were classified into negative $(-4 \sim-1)$, neutral (0) and positive (1 $\sim 4$ ) in this analysis.

TABLE 2

COUNTS OF COGNITIVE (SR) AND AFFECTIVE (FV) VALUE COMBINATIONS OVER THE FACIALLY EXPRESSIVE GROUP (17 RESPONDENTS)

\begin{tabular}{|c|c|c|c|c|}
\hline SR FV & $\begin{array}{c}\text { Positive } \\
\text { FV }\end{array}$ & $\begin{array}{c}\text { Neutral } \\
\text { FV }\end{array}$ & $\begin{array}{c}\text { Negative } \\
\text { FV }\end{array}$ & Sum \\
\hline Positive SR & 92 & 216 & $\mathbf{5 8}$ & 366 \\
\hline Neutral SR & 0 & 2 & 0 & 2 \\
\hline Negative SR & $\mathbf{2}$ & 61 & 79 & 142 \\
\hline Sum & 94 & $279^{2}$ & 137 & 510 \\
\hline
\end{tabular}

Table 2 shows the number of trials each combination happened during the experiments for the 17 respondents in the facially expressive group. In a between-subjects analysis, people showed more positive SRs (366 trials out of 510) than negative SRs (142 trials out of 510). The majority (13 out of 17 respondents) showed some negative FVs $(M=4.46$, $S D=2.93$, total 58 trials) even when they rated positive SRs. Thus, there were many times when a person's face expressed a negative experience, but they selected the answer on the questionnaire indicating it was positive. When people showed neutral FVs, they were more likely to give positive SRs (216 trials) than negative SRs (61 trials). Overall, people showed more negative FVs (137 trials out of 510) than positive FVs (94 trials out of 510). The large number of negative expressions lends evidence to the success of this protocol in enabling respondents to be comfortable making negative facial expressions to a computer.

\footnotetext{
${ }^{2}$ There was one trial during which outcome FV and evaluation FV had opposite valences with each other; thus, the trial was considered neutral FV value. This makes the total count of trials with neutral FV value $(=279)$ one more than the total count of trials with neutral outcome and evaluation FVs (=278 on Table 1$)$.
}

Cognitive values from self report (SR) and affective values from facial valence (FV), while correlated significantly (Pearson's $r=0.44, p<.001$ when excluding trials with neutral SR and/or neutral FV; $r=0.14, p<.01$ when including all), do not have high correlation value compared to the maximum possible value of $r=1.0$. Also, Kendall's $\tau=$ $0.45, p<.001$ when excluding all trials with neutral SR or neutral FV; and $\tau=0.09, p<.05$ when including all. Thus, while they agreed in the same general direction, they also carried different information. In particular, we found that the face tended to show more negative responses while selfreport showed more positive (in the group that was facially expressive); hence the two measures yield different information, supporting hypothesis $H 1$.

SR and FV responses for the most/least favored beverages: Of the 17 respondents in the facially expressive group, 7 favored StoreSoda and 10 favored NovelSoda in terms of their "after-the-test" (after 30 trials) preference. We wished to objectively evaluate how well the proposed measures forecast this after-the-test preference for each respondent. We computed the true positives (TP), the true negatives $(\mathrm{TN})$, the false positives (FP), and the false negatives of the $2 \times 2$ contingency table of response (positive, negative) $\times$ beverage (after-the-test most favored, after-the-test least favored) in terms of each measure (SR or FV) for each person in the facially expressive group (See Table 3).

TABLE 3

$2 \times 2$ CONTINGENCY TABLE OF RESPONSE (POSITIVE, NEGATIVE) $\times$ BEVERAGE (AFTER-THE-TEST MOST FAVORED, AFTER-THETEST LEAST FAVORED) IN TERMS OF EACH MEASURE (SR OR FV) FOR EACH PERSON

\begin{tabular}{|c|c|c|}
\hline & $\begin{array}{c}\text { After-the-test } \\
\text { Most favored } \\
\text { beverage }\end{array}$ & $\begin{array}{c}\text { After-the-test } \\
\text { Least favored bever- } \\
\text { age }\end{array}$ \\
\hline Positive response & TP (true positive) & FP (false positive) \\
\hline Negative response & FN (false negative) & TN (true negative) \\
\hline
\end{tabular}

Here, TP means the number of trials where the person showed positive responses for his or her after-the-test most favored beverage while FP is where the person showed positive responses for his or her after-the-test least favored beverage. Also, FN means the number of trials where the person showed negative responses for his or her after-the-test most favored beverage, while $\mathrm{TN}$ is where the person showed negative responses for his or her after-the-test least favored beverage. The TP rate (or sensitivity) is defined as TP/ (TP + $\mathrm{FN}$ ), and the $\mathrm{TN}$ rate (or specificity) as $\mathrm{TN} /(\mathrm{FP}+\mathrm{TN})$ [33].

Comparing the $\mathrm{TP}$ rates for each person in the facially expressive group, we found that the TP rate of the SR measure was significantly larger than that of the FV measure $(M=0.93$ vs. 0.67 ; within-subjects, $T(16)=2.56, p<.05)$. Comparing the TN rates, we found that the TN rate of the FV measure was significantly larger than that of the SR measure ( $M=0.88$ vs. 0.51 ; within-subjects, $T(16)=3.76, p$ $<.01)$. Also, pooling responses over the facially expressive group: $\mathrm{TP}=244, \mathrm{FN}=14, \mathrm{FP}=122, \mathrm{TN}=128$ (in the $\mathrm{SR}$ measure), while $\mathrm{TP}=75, \mathrm{FN}=22, \mathrm{FP}=19, \mathrm{TN}=115$ (in the FV measure). These findings are explained by the SR 
measure being biased toward positive responses and the FV measure biased toward negative responses. Overall people said very positive things about both products, but their facial expressions were much more negative. These complementary characteristics of SR and FV measures suggest that both the cognitive and affective measures have valuable contributions to make, and a combined measure of FV and SR may work best to discriminate the most/least favored beverages for each person.

Discriminability of SR and FV measures for the most/least favored beverages: For the discriminability analysis below, we computed the true positive rate $\equiv \mathrm{TP} /(\mathrm{TP}+\mathrm{FN})$ and the false positive rate $\equiv \mathrm{FP} /(\mathrm{FP}+\mathrm{TN})$. For facially non-expressive groups (where no positive or negative facial expressions occurred), $\mathrm{TP}=\mathrm{FN}=0$ or $\mathrm{FP}=\mathrm{TN}=$ 0 , we need to avoid dividing by zero and make sure we can still interpret $d_{\mathrm{FV}}^{\prime}$. In these cases, the easiest solution, since the facial expression valences are not contributing to any discriminability, is to assume the two rates are equal, giving the desired $d_{\mathrm{FV}}^{\prime}=0$. This assumption affects only Fig 4 in this paper, where we see the nonexpressive data lying on the horizontal axis.

It is worthwhile to note that $d_{\text {SR }}^{\prime}$, the discriminability using self-reports, of the facially non-expressive group $(\mathrm{N}=17)$ is significantly smaller than that of the facially expressive group $(\mathrm{N}=17)(M=1.14$ vs. 2.18 ; between-subjects, $T(32)=-$ $1.86, p<.05)$. In terms of the absolute difference between the average cognitive values of two beverages $\left|\operatorname{Diff}_{\mathrm{SR}}\right|$, the facially non-expressive group showed a significantly smaller $\mid$ Diff $_{\mathrm{SR}} \mid$ than the facially expressive group $(M=0.78$ vs. 1.62 ; between-subjects, $T(32)=-2.72, p<.01)$. Moreover, within the facially non-expressive group (17 persons), there were two clusters: 10 respondents had very small $d_{\mathrm{SR}}^{\prime}(M=0.12$, $S D=0.06)$ and $\left|D i f f_{\mathrm{SR}}\right|(M=0.44, S D=0.21)$, and the other 7 respondents had higher $d_{\mathrm{SR}}^{\prime}(M=2.60, S D=0.75)$ and $\left|D i f f_{\mathrm{SR}}\right|$ $(M=1.26, S D=0.98)$. That is, the facially non-expressive group can be decomposed into two classes as follows:

- low $d_{\mathrm{SR}}^{\prime} \&$ low $d_{\mathrm{FV}}^{\prime}(\mathrm{N}=10)$ : hardly discriminable by $\mathrm{FV}$ or by SR

- high $d_{\mathrm{SR}}^{\prime} \&$ low $d_{\mathrm{FV}}^{\prime}(\mathrm{N}=7)$ : hardly discriminable by $\mathrm{FV}$, but easily by SR

Keeping in mind that $d_{\mathrm{FV}}^{\prime}$, the discriminability of $\mathrm{FV}$, is essentially zero for this facially non-expressive group, the findings imply that there exists a group of people (10 out of 34 respondents) whose beverage preference is very difficult to discern in both SR and FV measures.

Fig. 4 shows the relationship between $d_{\mathrm{SR}}^{\prime}$ and $d_{\mathrm{FV}}^{\prime}$ over the facially expressive $(\mathrm{N}=17)$ and facially non-expressive $(\mathrm{N}=17)$ groups. Each dot in this figure represents a respondent's $d_{\mathrm{SR}}^{\prime}$ and $d_{\mathrm{FV}}^{\prime}$. The two groups above correspond to the two clusters positioned on the horizontal axis in Fig. 4.

We also grouped the respondents into three classes (also shown in Fig 4) for the facially expressive group as follows: For the facially expressive $\mathrm{N}=17$, we evaluated the $d^{\prime}$ SR-toFV ratio $\left(d_{\mathrm{SR}}^{\prime} / d_{\mathrm{FV}}^{\prime}\right)$ for each person and observed three clusters according to the $d^{\prime}$ SR-to-FV ratio: a high $d^{\prime}$ ratio group $\left(d_{\mathrm{SR}}^{\prime} / d_{\mathrm{FV}}^{\prime} \geq 1.4\right)$, a medium $d^{\prime}$ ratio group $\left(d_{\mathrm{SR}}^{\prime} / d_{\mathrm{FV}}^{\prime}\right.$ $<1.4$ and $\left.d_{\mathrm{SR}}^{\prime} / d_{\mathrm{FV}}^{\prime}>0.7\right)$, and a low $d^{\prime}$ ratio group $\left(d_{\mathrm{SR}}^{\prime} /\right.$
$\left.d_{\mathrm{FV}}^{\prime} \leq 0.7\right)(N=3$ vs. 7 vs. $7 ; M=5.83$ vs. 1.01 vs. 0.17 ; between-subjects, $F(16)=26.6, p<.001)$. For the high $d^{\prime}$ ratio group, $d_{\mathrm{SR}}^{\prime}>d_{\mathrm{FV}}^{\prime}(M=3.01$ vs. 0.53 ; within-subjects, $T(2)=2.61, p<.05)$; for the medium $d^{\prime}$ ratio group, $d_{\mathrm{SR}}^{\prime} \approx d_{\mathrm{FV}}^{\prime}$ ( $M=3.62$ vs. 3.57 ; within-subjects, $T(6)=0.18, N S)$; for the low $d^{\prime}$ ratio group, $d_{\mathrm{SR}}^{\prime}<d_{\mathrm{FV}}^{\prime}(M=0.39$ vs. 2.23 ; withinsubjects, $T(6)=-3.76, p<.01)$. That is, three classes for the facially expressive group are:

- high $d^{\prime}$ ratio $\left(d_{\mathrm{SR}}^{\prime} / d_{\mathrm{FV}}^{\prime}\right)$ group $(\mathrm{N}=3)$

- medium $d^{\prime}$ ratio $\left(d_{\mathrm{SR}}^{\prime} / d_{\mathrm{FV}}^{\prime}\right)$ group $(\mathrm{N}=7)$

- low $d^{\prime}$ ratio $\left(d_{\mathrm{SR}}^{\prime} / d_{\mathrm{FV}}^{\prime}\right)$ group $(\mathrm{N}=7)$

If SR and FV told us the same thing, we would expect that $d_{\mathrm{SR}}^{\prime}$ and $d_{\mathrm{FV}}^{\prime}$ would be highly correlated but in fact $d_{\mathrm{SR}}^{\prime}$ and $d_{\mathrm{FV}}^{\prime}$ are not significantly correlated (Pearson's $r=0.39, p>$ $.05)$. This supports Hypothesis $H 2$.

Our findings also have an important practical implication. First, the more people a company has to recruit for a study, the higher the cost. Second, companies in several industries have told us that they routinely have to inflate the number of respondents, very high, in order to get significant discrimination when comparing two products that are both similar, e.g. if both are "likeable" in self-reports (SR). We found, for the two very similar products here, that combining the SR and FV measures increases discriminability such that fewer respondents are required to predict customers' overall preference and the market success/failure of products for the same level of accuracy. In short, improving the overall $d^{\prime}$ means reducing the overall cost to a business, and ultimately that can translate into customer savings.

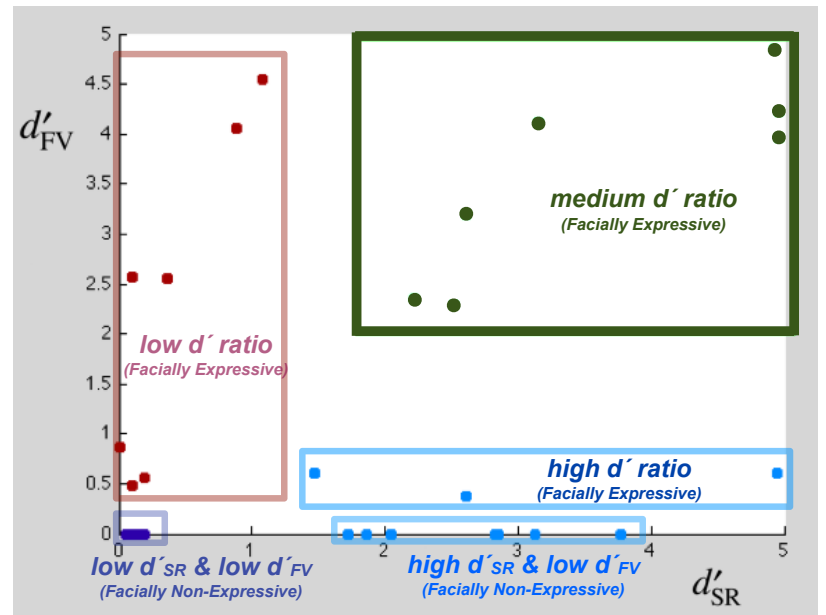

Fig. 4. Discriminability of facial valence vs. discriminability of selfreport over the facially expressive $(\mathrm{N}=17)$ and facially non-expressive $(\mathrm{N}=17)$ groups (each dot corresponds to a respondent based on discriminating their after-the-test beverage preference.)

\subsection{Analysis 2: Predictions of Market Success/Failure of Beverages}

In order to maximize ecological validity of our experiment we tested two real products with known outcomes in the marketplace: StoreSoda is a FamousBrand successful beverage while NovelSoda was an attempt by FamousBrand to make another successful beverage, but it did not succeed after it went to the marketplace. We wished to evaluate how well the affective and cognitive measures from individuals in our test predict the actual marketplace results for both 
products. Our hypotheses are:

H3: Cognitive and affective measures have different properties in predicting market success/failure and integrating cognitive and affective measures can improve the predictability of success/failure.

Since affective wanting may motivate or drive people to seek out the product for purchase, and thus potentially increase marketplace sales, we also hypothesize:

H4: The measure of affective wanting is more critical than that of affective liking in making accurate market success/failure predictions.

In this section we propose six predictive models using different combinations of affective and cognitive measures. We evaluate these models in terms of nine performance criteria. We observe that the model combining both cognitive value (SR) and affective wanting (Outcome FV) measures performs the best overall. One potential criticism of our analysis might be overfitting or retrofitting to known outcomes. It is important to underscore that in our work this criticism does not apply because we did not use the known outcome information in building any of the six models. We did not train any of these models on the data. That is, there are no model parameters in each model that were fitted by leveraging the known outcomes. All of the models would perform exactly as they did here whether or not the market outcomes were known.

SR and FV measures to predict market success/failure of beverages: We use actual market success/failure data for both sodas to test hypotheses $H 3$ and $H 4$. Taking into account that StoreSoda performed in the top 25\% (success) and NovelSoda performed in the lower $40 \%$ (failure) in the market, in this analysis, the market successful beverage indicates StoreSoda and unsuccessful indicates NovelSoda. Here we examine all the measures not for the most/least favored beverage as in the last section, but rather, for market successful/unsuccessful beverages. We computed the TP, TN, $\mathrm{FP}$, and FN of the $2 \times 2$ contingency table of response (positive, negative) $\times$ beverage (market successful, market unsuccessful) in terms of each measure (SR or FV) for each person in the facially expressive group (See Table 4).

TABLE 4

$2 \times 2$ CONTINGENCY TABLE OF RESPONSE (POSITIVE, NEGATIVE) $\times$ BEVERAGE (MARKET SUCCESSFUL, MARKET UNSUCCESSFUL) IN TERMS OF EACH MEASURE (SR OR FV) FOR EACH RESPONDENT

\begin{tabular}{|c|c|c|}
\hline & $\begin{array}{c}\text { Market Successful } \\
\text { beverage }\end{array}$ & $\begin{array}{c}\text { Market Unsuccess- } \\
\text { ful beverage }\end{array}$ \\
\hline Positive response & TP (true positive) & FP (false positive) \\
\hline Negative response & FN (false negative) & TN (true negative) \\
\hline
\end{tabular}

To evaluate the effectiveness of the market success predictions of a SR or FV measure we evaluated several performance statistics from the contingency table of responses (positive, negative) $\times$ beverages (market successful, market unsuccessful). Specifically, we examine how the perform- ance statistics vary over the facially expressive and/or the whole groups when the SR values alone, the FV values alone, or the SR and FV combined values are used for the market success prediction. Because our method and measures are new, we also compare against all of the following well-known criteria [33],[34],[35]:

- Positive predictive value $=\mathrm{TP} /(\mathrm{TP}+\mathrm{FP})$

- Negative predictive value $=\mathrm{TN} /(\mathrm{FN}+\mathrm{TN})$

- Likelihood ratio positive (LR+)

$=$ sensitivity / $(1-$ specificity $)$

$=($ true positive rate $) /($ false positive rate $)$

$=(\mathrm{TP} /(\mathrm{TP}+\mathrm{FN})) /(\mathrm{FP} /(\mathrm{FP}+\mathrm{TN}))$

- Likelihood ratio negative (LR-)

$=(1-$ sensitivity $) /$ specificity

$=($ false negative rate $) /$ (true negative rate $)$

$=(\mathrm{FN} /(\mathrm{TP}+\mathrm{FN})) /(\mathrm{TN} /(\mathrm{FP}+\mathrm{TN}))$

- Accuracy $=(\mathrm{TP}+\mathrm{TN}) /(\mathrm{TP}+\mathrm{FN}+\mathrm{FP}+\mathrm{TN})$

- MCC (Matthews correlation coefficient)

$=(\mathrm{TP} \times \mathrm{TN}-\mathrm{FP} \times \mathrm{FN}) / \sqrt{ }((\mathrm{TP}+\mathrm{FP})(\mathrm{TP}+\mathrm{FN})(\mathrm{TN}+$ $\mathrm{FP})(\mathrm{TN}+\mathrm{FN}))$

- $d^{\prime}=Z$ (sensitivity) $-Z(1-$ specificity) where $Z(p), p \in[0,1]$ is the inverse of the standard cumulative normal distribution

The LR criteria are of interest in the following way: First, it is desirable that LR+ should be high and LR- should be low; these are comparable to desiring both high sensitivity and high specificity. The LR+ is the likelihood that a given positive response of a measure would be expected in a market successful beverage compared to the likelihood that the same response would occur in a market unsuccessful beverage. The LR- would be the likelihood that a given negative response would occur in a market successful beverage compared to the likelihood that it occurs in a market unsuccessful beverage.

Table 5 shows the results for the following six models:

- (Model 1) [Cog Val (N=39)] SR (cognitive value) over all respondents

- (Model 2) [Cog Val (N=17)] SR (cognitive value) alone over all the facially expressive respondents

- (Model 3) [Aff Val (N=17)] FV (affective value) alone over all the facially expressive respondents

- (Model 4) [Cog Val \& Aff Val (N=17)] SR \& FV (cognitive value $\&$ affective value) combined over all the facially expressive respondents

- (Model 5) [Aff Want $(\mathrm{N}=17)$ ] Outcome FV (affective wanting value) alone over all the facially expressive respondents

- (Model 6) [Cog Val \& Aff Want $(\mathrm{N}=17)]$ SR \& Outcome FV (cognitive value \& affective wanting value) combined over all the facially expressive respondents

Note that Models 4 and 6 combine affective and cognitive measures. Discussion follows below. For Model 1 using SR alone over all respondents, the overall sensitivity of selfreport for predicting market success/failure is high while the specificity and the discriminability $d$ ' of self-report are very low. For Models 2-6, we consider only the facially expressive group ( 7 StoreSoda favorers and 10 NovelSoda favorers in terms of after-the-test beverage preference). The FV alone (Model 3) does not do as well on sensitivity as the SR alone 
TABLE 5

THE MARKET SUCCESS PREDICTION PERFORMANCE OF DIFFERENT MODELS IN TERMS OF MULTIPLE CRITERIA. THE BEST RESULT IN EACH ROW IS PRINTED IN BOLD FACE TYPE. NOTE THAT \pm VALUES REPRESENT STANDARD ERRORS OF THE MEAN.

\begin{tabular}{|c|c|c|c|c|c|c|}
\hline $\begin{array}{l}\text { Model } \\
\text { (Respondents) }\end{array}$ & $\begin{array}{l}\text { 1. Cog Val } \\
(\mathrm{N}=39)\end{array}$ & $\begin{array}{l}\text { 2. Cog Val } \\
\qquad(\mathrm{N}=17)\end{array}$ & $\begin{array}{l}\text { 3. Aff Val } \\
\qquad(\mathrm{N}=17)\end{array}$ & $\begin{array}{l}\text { 4. Cog Val \& } \\
\text { Aff Val } \\
(\mathrm{N}=17)\end{array}$ & $\begin{array}{l}\text { 5. Aff Want } \\
\qquad(\mathrm{N}=17)\end{array}$ & $\begin{array}{l}\text { 6. Cog Val \& } \\
\text { Aff Want } \\
(\mathrm{N}=17)\end{array}$ \\
\hline Measures & $\mathrm{SR}$ & SR & FV & SR \& FV & Outcome FV & SR \& Outcome FV \\
\hline $\mathrm{TP}$ & 484 & 201 & 54 & 52 & 34 & 32 \\
\hline FP (lower is better) & 464 & 165 & 40 & 40 & 24 & 24 \\
\hline FN (lower is better) & 100 & 56 & 60 & 28 & 25 & 8 \\
\hline $\mathrm{TN}$ & 107 & 86 & 77 & 51 & 39 & 33 \\
\hline Sensitivity & $0.83 \pm 0.002$ & $0.78 \pm 0.005$ & $0.47 \pm 0.007$ & $0.65 \pm 0.010$ & $0.58 \pm 0.012$ & $0.80 \pm 0.010$ \\
\hline Specificity & $0.19 \pm 0.002$ & $0.34 \pm 0.007$ & $0.66 \pm 0.007$ & $0.56 \pm 0.010$ & $0.62 \pm 0.010$ & $0.58 \pm 0.012$ \\
\hline PPV & $0.51 \pm 0.002$ & $0.55 \pm 0.005$ & $0.57 \pm 0.007$ & $0.57 \pm 0.007$ & $0.59 \pm 0.012$ & $0.57 \pm 0.012$ \\
\hline NPV & $0.52 \pm 0.003$ & $0.61 \pm 0.010$ & $0.56 \pm 0.007$ & $0.65 \pm 0.010$ & $0.61 \pm 0.010$ & $0.80 \pm 0.010$ \\
\hline $\mathrm{LR}+$ & $1.02 \pm 0.002$ & $1.19 \pm 0.016$ & $1.40 \pm 0.046$ & $1.50 \pm 0.043$ & $1.54 \pm 0.067$ & $1.92 \pm 0.067$ \\
\hline LR- (lower is better) & $0.92 \pm 0.011$ & $0.64 \pm 0.002$ & $0.80 \pm 0.018$ & $0.63 \pm 0.024$ & $0.70 \pm 0.027$ & $0.35 \pm 0.023$ \\
\hline Accuracy & $0.51 \pm 0.001$ & $0.57 \pm 0.005$ & $0.57 \pm 0.007$ & $0.60 \pm 0.008$ & $0.60 \pm 0.009$ & $0.67 \pm 0.009$ \\
\hline $\mathrm{MCC}$ & $0.02 \pm 0.003$ & $0.14 \pm 0.011$ & $0.13 \pm 0.013$ & $0.21 \pm 0.015$ & $0.19 \pm 0.020$ & $0.38 \pm 0.018$ \\
\hline$d^{\prime}$ & $0.06 \pm 0.008$ & $0.37 \pm 0.029$ & $0.34 \pm 0.034$ & $0.54 \pm 0.041$ & $0.49 \pm 0.051$ & $1.05 \pm 0.058$ \\
\hline
\end{tabular}

(Model 2); however, it does better on specificity and on discriminability $d^{\prime}$. Also, the FV alone (Model 3) has a higher $\mathrm{LR}+$ than the SR alone (Model 2), and the SR alone (Model 2) has a lower LR- than the FV alone (Model 3), reflecting the tendency of SR to be positive and FV to be negative for these beverage tests. Before we discuss Models 4-6, recall that our goal all along has been to assess what the different affective, behavioral, and cognitive measures tell us not only separately, but to see if they offer something more powerful together than traditional cognitive-only measures provide. We are particularly interested in building a measure that is more reliable and trustworthy than what has been available in the past. Reliability and trustworthiness are enhanced by consistency; therefore, we predict that if a person's affective and cognitive measures are consistent, then they will be more reliable predictors. For Model 4, we take only the samples where FV and SR are congruent. In other words, the new model only considers samples of \{positive FV \& positive SR \} or \{negative FV \& negative SR .

The combined SR \& FV model (Model 4), based on the consistent data, provides higher d', higher LR+, lower LR-, higher accuracy and higher MCC than the SR model or the FV model. In other words, Model 4's combined cognitive and affective value represents respondents' beverage preference better given these four popular criteria (LR+, LR-, Accuracy, MCC). Also, it finds that respondents like StoreSoda more than NovelSoda, predicting that StoreSoda would be more successful than NovelSoda in the real marketplace, which is consistent with the actual market selling success/failure of the two products. Thus, combining consistent affective and cognitive measures is more predictive of the market success/failure, supporting Hypothesis $H 3$.

We are interested in seeing whether affective wanting (outcome FV, i.e., satisfaction/disappointment) and affective liking (evaluation FV, i.e., liking/disliking) differ in their ability to predict market success/failure. The results below show the nrediction nerformances of the outcome FV model alone (Model 5) and the combined SR \& outcome FV model (Model 6). For Model 5, we only use outcome FV (affective wanting) without using evaluation FV (affective liking). For Model 6, we employ the congruent responses between SR and outcome FV (cognitive value and affective wanting) for each beverage.

Clearly the outcome FV model (affective wanting, Model 5 ) is significantly better than the FV model (affective wanting \& affective liking, Model 3), and the SR \& outcome FV model (cognitive value \& affective wanting, Model 6) is significantly better than SR \& FV model (cognitive value \& affective value, Model 4) on the different criteria of prediction performance. Thus, in this study, affective wanting measures were more effective than affective liking measures in predicting the customers' overall preference, supporting Hypothesis $H 4$.

Figs. 5, 6, 7, 8 and 9 summarize the different criteria for all six models above, as well as four additional ones $(\mathrm{N}=17)$ : cognitive wanting alone, cognitive liking alone, affective liking alone, and cognitive value coupled with affective liking. These comparisons allowed us to answer, for this set of real-world data, whether liking or wanting was more important. For these data, the combination of affective wanting (from the face) with cognitive value (from self-report), Model 6, strongly outperformed all the other combinations.

We cannot claim based on this one study that the cognitive value and affective wanting combination will always outperform the other measures in general. We, however, showed that this was a robust measure in this context without training models on any known outcomes. This combination gave the best performance for the majority of performance criteria, without any tuning on this data. Most importantly, we see that combining affective measures and cognitive measures gives a significant performance improvement over the current standard of use of only cognitive measures. Because the cognitive measures also asked respondents' 
about their feelings, this boost suggests that the objective affective measures from facial valence provide value over self-reported feelings. This paper introduces the new framework involving both cognitive and affective measures, identifying strengths of each. We hope that in the future other researchers can apply the exact or a similar framework to replicate our findings in this paper. In the future, as more product data sets are obtained, the model parameters may also be trained on the data, and tuned to optimize future generalization performance.

\section{CONCLUSION AND Discussion}

Market researchers have commonly experienced that people said (self-report) they loved the product, and yet it still flopped in the marketplace. We do not think respondents intend to mislead marketers; instead, we assume respondents want to communicate their true feelings about products and want to see better products hit the market, but current market research practices have not made accurate communication of emotion easy.

Most standard practices today do not yet pay careful attention to principles of reliable and comfortable affective elicitation and communication: They do not give customers an adequate number of trials with the product to get to know how they feel about the product, a process that can naturally involve complex changing feelings. They also assume people know how to reliably map their momentary feeling to a simple digit, as if feelings about an experience really were reducible to a single number. They also often have an experimenter present observing, making it socially awkward to express negative feelings, etc.

With these challenging realities in mind we constructed a new framework to make it easier, more comfortable and more reliable to elicit and measure respondents' intuitive feelings about products. The $\mathrm{ABC}$ framework provides a multiple-trial random-outcome protocol to easily and naturally elicit respondents' feelings, and the multi-modal measures can unveil more about customer affective, behavioral, and cognitive preference and increase the reliability of market success prediction. An important contribution of this work is an entirely new methodology to elicit and capture affective wanting as an "outcome facial valence". We evalu ated the new affective measures along with traditional cogni-

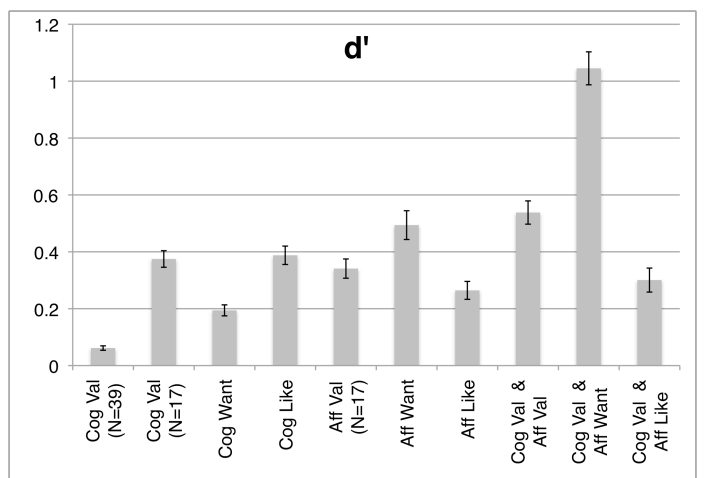

Fig. 5. Discriminability, $d^{\prime}$, for all the affective and cognitive measures singly and in combination. [Cog Val \& Aff Want] is significantly higher than all the others. Error bars represent standard errors of the mean.

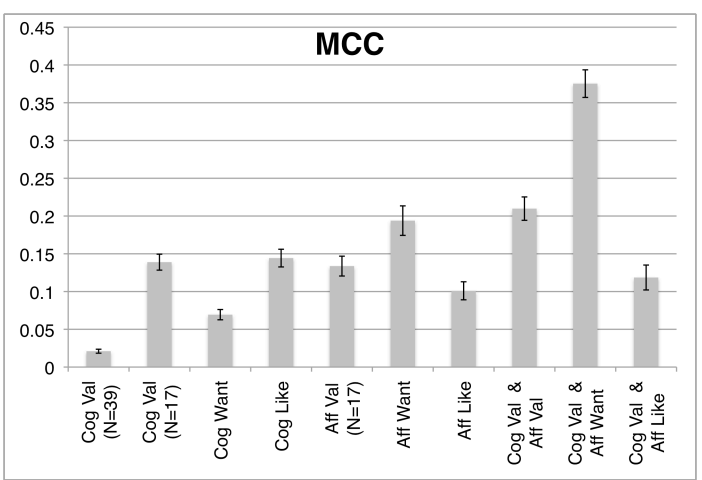

Fig. 6. MCC, for all the affective and cognitive measures singly and in combination. Error bars represent standard errors of the mean.

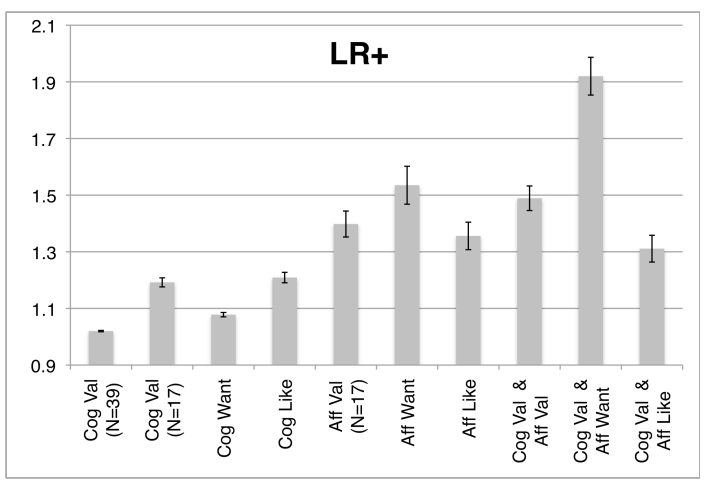

Fig. 7. LR+, for all the affective and cognitive measures singly and in combination. Error bars represent standard errors of the mean.

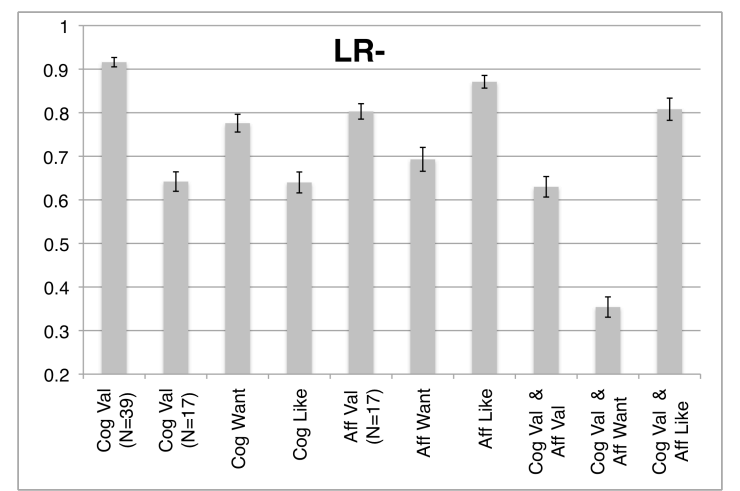

Fig. 8. LR-, for all the affective and cognitive measures singly and in combination. Lower is better. Error bars represent standard errors of the mean.

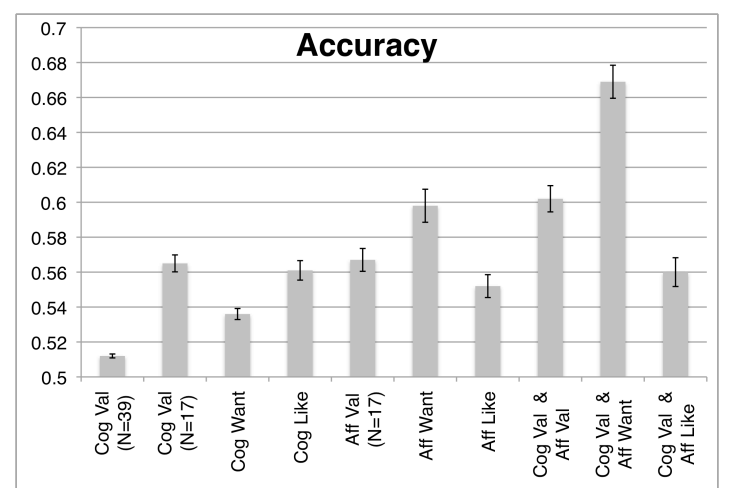

Fig. 9. Accuracy, for all the affective and cognitive measures singly and in combination. Error bars represent standard errors of the mean. 
tive and behavioral measures. As a cost-advantage, this study involved a much smaller group than the large samples usually required in order to obtain significant power when only self-report measures are used. With only $\mathrm{N}=39$ it provided statistically significant discrimination and new insights. Compared to using the cognitive measure only, the combined cognitive-affective measure enables companies to figure out the actual preferences of more respondents out of the total respondents. Thus, companies can reduce the number of respondents in making a prediction with the same confidence level. Or, companies can significantly increase the confidence level in their prediction without significant cost to their experiment budget.

Our study confirmed the difficulty of obtaining significant discrimination based only on cognitive self-report measures when two products are similar: self-report measures were overall very positive for both sweet cola beverages, and choices of favorite beverage were fairly evenly split in preferring the StoreSoda or the NovelSoda, with only very slight preference for StoreSoda (analyzing the questionnaire data, the ratios were: 20:18). Behaviorally, people consumed the same amount of StoreSoda and NovelSoda per sip and there was a subtle bias toward choosing the machine more likely to give NovelSoda: $54.6 \%$ of the time it was chosen over the machine more likely to give StoreSoda. On balance, cognitive and behavioral measures showed the two products to be preferred nearly equivalently.

Combining all of the cognitive (questionnaire) items and behavioral items would suggest that NovelSoda should have had a great chance to succeed in the marketplace - to perform as well as StoreSoda. Of course this is a small sample, it is only one regional area, and it does not take into account other factors such as the packaging, marketing or possible effect of the weather or season. Nonetheless, this finding from our study is important because it shows that surveying this sample of respondents could have led to the same decision that was chosen historically by FamousBrand: they took NovelSoda to market, investing a substantial amount of money, time, transportation resources (fuel for trucks, etc) and other natural resources to package, distribute, and promote the product, and ultimately more unplanned resources to physically truck it back from the store shelves. Based on cognitive and behavioral data, going to market appeared to be a reasonable decision; however, this result is only part of the story, as the results of this study suggest.

We found that respondents showed significantly more negative FVs than positive FVs for NovelSoda than for StoreSoda. People's negative affective feelings that could influence their purchases showed up in FV measures and not in the self-reported cognitive or in the behavioral measures. In particular, the "outcome" facial valences (affective wanting values) that were shown when a machine told them which product to sip - reflecting a new measure of customer wanting through facial expressions of satisfaction or disappointment - showed the highest discriminability $\left(d^{\prime}\right)$ when combined with cognitive self-report. Meanwhile, self-report did not provide high discriminability, and tended to be biased to say positive things.
We have shown that the measures presented here are capable of significantly enhancing discriminability even among two similar real-world products that people say they like nearly equally. Our experimental results suggest that combining the SR and FV measures in customer research on experience/hedonic products can require fewer respondents to predict customers' overall preference and the market success/failure of products for the same level of accuracy. In addition, since tastes may change in our culture over time as well, we think it is important to reduce the number of respondents required for obtaining any statistically meaningful conclusions for market prediction so such tests can be made more easily and more often.

Our framework can be applied in many different contexts in which a mechanism to predict future market success/failure of new products is needed - the approach could be easily applied to any product or service that can be consumed or used such that facial expressions can be recorded or observed, or for any product concept or service delivered with a computer in front of a person, including over the Internet or in a kiosk. Our framework, as demonstrated here, can also be applied within standard A/B testing practice. Thus, it is not limited to beverages.

People's buying decisions and the market outcomes can be dramatically influenced by the revelation of branding information. Although the paper focuses on the framework in blind taste tests, this framework itself can be extended and generalized to include other contexts such as brands or commercials. Combining the affective and cognitive measure might remain very effective in predicting market outcomes from the experiment setting involving other context stimuli. The key in our framework is to comfortably and repeatedly elicit affective, behavioral, and cognitive components, recognizing that they all are pieces of the human experience, and when properly combined, they provide powerful new insights into how to make experiences better.

\section{ACKNOWLEDGEMENTS}

The authors wish to thank the respondents for participating in this study. We also acknowledge the generous support of MIT Media Lab consortium sponsors for this work.

\section{REFERENCES}

[1] D. Kahneman, "Experienced Utility and Objective Happiness: A Moment-Based Approach," Choices, values, and frames, pp. 673692, 2000.

[2] R. J. Larsen, and B. L. Fredrickson, "Measurement issues in emotion research," Well-being: The foundations of hedonic psychology, D. Kahneman, Diener Ed and N. Schwarz, eds., pp. 4060, New York: Russell Sage Foundation, 1999.

[3] D. M. Stayman, and D. A. Aaker, "Continuous Measurement of Self-Report of Emotional Response,” Psychology \& Marketing, vol. 10, no. 3, pp. 199-214, 1993.

[4] D. Kahneman, P. P. Wakker, and R. Sarin, "Back to Bentham? Explorations of Experienced Utility," The Quarterly Journal of Economics, vol. 112, no. 2, pp. 375-405, 1997.

[5] M. Koenigs, and D. Tranel, "Prefrontal cortex damage abolishes brand-cued changes in cola preference," Social Cognitive and 
Affective Neuroscience, vol. 3, no. 1, pp. 1-6, 2008.

[6] M. Gladwell, Blink: The power of thinking without thinking, New York: Little, Brown and Company, 2005.

[7] http://en.wikipedia.org/wiki/New_Coke

[8] J. I. Davis, A. Senghas, and K. N. Ochsner, "How does facial feedback modulate emotional experience?," Journal of research in personality, vol. 43, no. 5, pp. 822-829, 2009.

[9] R. S. Sutton, and A. G. Barto, Reinforcement Learning: An Introduction: MIT Press, 1998.

[10] J. B. Cohen, M. T. Pham, and E. B. Andrade, "The Nature and Role of Affect in Consumer Behavior," Handbook of Consumer Psychology, Curtis P. Haugtvedt, Paul Herr, and Frank Kardes (Eds.), 2006.

[11] K. C. Berridge, and J. W. Aldridge, "Decision utility, incentive salience, and cue-triggered "wanting'," Oxford Handbook of Human Action, E. Morsella, Bargh, J.A., \& Gollwitzer, P.M. (Eds.), ed., pp. 509-532: Oxford University Press, 2009.

[12] K. C. Berridge, and T. E. Robinson, "Parsing reward," Trends Neurosci, vol. 26, no. 9, pp. 507-13, 2003.

[13] G. Loewenstein, and J. S. Lerner, "The role of affect in decision making," Handbook of affective science, H. G. R. Davidson, \& K. Scherer (Eds.), ed., pp. 619-642, Oxford: Oxford University Press, 2003.

[14] J. Panksepp, Affective Neuroscience: The Foundations of Human and Animal Emotions: Oxford University Press, 1998.

[15] J. Panksepp, "Are emotions more than learned behaviors?," Trends in cognitive sciences, vol. 10, no. 3, pp. 96-97, 2006.

[16] P. Ekman, and W. V. Friesen, Facial action coding system: A technique for the measurement of facial movement: Consulting Psychologists Press, Palo Alto, CA, 1978.

[17] J. A. Russell, “A circumplex model of affect," Journal of Personality and Social Psychology, vol. 39, no. 6, pp. 1161-1178, 1980.

[18] D. H. McBurney, and T. L. White, Research methods: Wadsworth, 2009.

[19] K. C. Berridge, "Pleasure, pain, desire, and dread: Hidden core processes of emotion," Well-being: The foundations of hedonic psychology, D. Kahneman, Diener Ed and N. Schwarz, eds., pp. 525-557, New York: Russell Sage Foundation, 1999.

[20] B. W. Balleine, and A. Dickinson, "Goal-directed instrumental action: contingency and incentive learning and their cortical substrates," Neuropharmacology, vol. 37, no. 4-5, pp. 407-19, 1998.

[21] A. Bechara, H. Damasio, D. Tranel et al., "Deciding Advantageously Before Knowing the Advantageous Strategy," Science, vol. 275, no. 5304, pp. 1293, 1997.

[22] K. C. Berridge, "Motivation concepts in behavioral neuroscience," Physiology and Behavior, vol. 81, no. 2, pp. 179-209, 2004.

[23] N. D. Daw, Y. Niv, and P. Dayan, "Uncertainty-based competition between prefrontal and dorsolateral striatal systems for behavioral control," Nature Neuroscience, vol. 8, no. 12, pp. 1704-1711, 2005.

[24] P. Dayan, Y. Niv, B. Seymour et al., "The misbehavior of value and the discipline of the will," Neural Networks, vol. 19, no. 8, pp. 1153-1160, 2006.

[25] D. Kahneman, "Maps of Bounded Rationality: Psychology for Behavioral Economics," The American Economic Review, vol. 93, nก 5 nn 1449-1475 2003
[26] G. Loewenstein, and T. O'Donoghue, "Animal Spirits: Affective and Deliberative Processes in Economic Behavior," Social Science Research Network, 2004.

[27] A. B. Satpute, and M. D. Lieberman, "Integrating automatic and controlled processes into neurocognitive models of social cognition," Brain Research, vol. 1079, no. 1, pp. 86-97, 2006.

[28] S. Baron-Cohen, S. Wheelwright, J. Hill et al., "The "Reading the Mind in the Eyes" Test revised version: a study with normal adults, and adults with Asperger syndrome or high-functioning autism," The Journal of Child Psychology and Psychiatry and Allied Disciplines, vol. 42, no. 02, pp. 241-251, 2001.

[29] G. Oliver, and J. Wardle, "Perceived Effects of Stress on Food Choice," Physiology \& Behavior, vol. 66, no. 3, pp. 511-515, 1999.

[30] D. A. Zellner, S. Loaiza, Z. Gonzalez et al., "Food selection changes under stress," Physiology \& Behavior, vol. 87, no. 4, pp. 789-793, 2006.

[31] T. D. Wickens, Elementary signal detection theory: Oxford University Press, USA, 2002.

[32] D. A. Kenny, Statistics for the social and behavioral sciences: Little, Brown Boston, 1987.

[33] T. Fawcett, "An introduction to ROC analysis," Pattern recognition letters, vol. 27, no. 8, pp. 861-874, 2006.

[34] D. G. Altman, and J. M. Bland, "Diagnostic tests 2: Predictive values," BMJ: British Medical Journal, vol. 309, no. 6947, pp. 102, 1994.

[35] P. Baldi, S. Brunak, Y. Chauvin et al., "Assessing the accuracy of prediction algorithms for classification: an overview," Bioinformatics, vol. 16, no. 5, pp. 412, 2000.

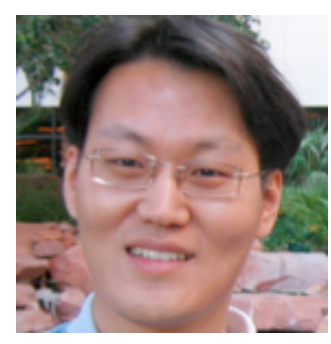

Hyung-il Ahn is a research staff member in IBM Research - Almaden. He completed his $\mathrm{PhD}$ in Media Arts and Sciences at the MIT Media Lab, affective computing group in 2010. He holds a Masters degree (2004) in Mechanical Engineering from MIT, and a Bachelors degree (1998) in Aerospace Engineering with highest honors from Seoul National University in South Korea. His doctoral research focused on the computational model of human behavioral, affective and cognitive decision making, inspired by findings in psychology, economics, neuroscience and machine learning. He has published broadly in the areas of affective computing, social media, human computer interaction, data analytics and machine learning. Contact him athiahn@us.ibm.com.

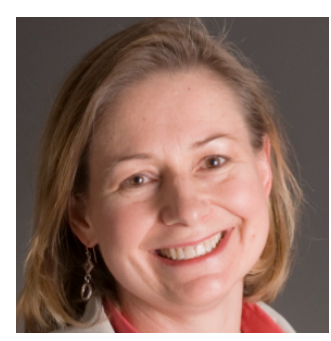

Rosalind W. Picard is a fellow of the IEEE and member of the IEEE Computer Society, is Professor of Media Arts and Sciences at the MIT Media Lab, founder and director of the Affective Computing Group, and co-founder of Empatica Inc, and Affectiva, Inc.

Her current research interests focus on the development of technology to help people comfortably and respectfully measure and communicate affective information, as well as on the development of models of affect that improve decision-making and learning. Picard has a Bachelor's in Electrical Engineering from Georgia Tech, and Masters and Doctorate of Science degrees in Electrical Engineering and Computer Science from the Massachusetts Institute of Technology. Contact her at picard@media.mit.edu. 